\title{
Tribenoside-lidocaine combination in wound healing of hemorrhoids: a review of literature
}

\begin{abstract}
Background: Hemorrhoids are one of the most common anorectal disease observed in clinical practice. Symptomatic hemorrhoids can be effectively treated with a pharmacological approach, particularly with topical drugs, associated with dietary and lifestyle changes.
\end{abstract}

\begin{abstract}
Aim: The aim of this review is to assess the effectiveness of tribenoside $5 \%+$ lidocaine $2 \%$ combination cream and suppository forms to manage the acute symptomatology of patients with hemorrhoids.

Method: Literatures were retrieved by a PubMed search, using different combinations of pertinent keywords (e.g., wound healing, tribenoside, hemorrhoids), without any limitations in terms of publication date and language. Papers which assessed the therapeutic efficacy and tolerability of tribenoside + lidocaine combination in patients with hemorrhoids were selected for inclusion according to their relevance for the topic, as judged by the authors.

Results: Five studies compared tribenoside + lidocaine combination with each of its single components: in 3 studies tribenoside + lidocaine was compared with lidocaine and in 2 studies with tribenoside. Moreover in 6 studies tribenoside + lidocaine has been compared with a corticosteroid containing preparation and 2 studies evaluated the product effectiveness in women with hemorrhoids, as a consequence of pregnancy or delivery. The results of all studies showed that the association tribenoside + lidocaine was significantly superior to lidocaine alone and had a similar clinical efficacy compared to tribenoside and corticosteroids. However, the tribenoside + lidocaine showed a faster relief of subjective symptomatology, than tribenoside and corticosteroids, with the advantage to avoid the possible steroid-related side-effects. Tribenoside + lidocaine has been well tolerated.
\end{abstract}

Conclusion: In conclusion, tribenoside + lidocaine represents a rapid, effective and safe option for treatment of hemorrhoids, and should be recommended as first-line treatment of this disease in clinical practice for its long-lasting healing effects on hemorrhoids with high efficacy and good tolerability.

Keywords: tribenoside, lidocaine, hemorrhoids, wound healing

\author{
Volume 9 Issue 6 - 202 I
}

\author{
Gokhan Faikoglu,' Kubra Saygisever- \\ Faikoglu, ${ }^{2}$ Fatmanur Otmar Ozcan, ${ }^{3}$ Barkin \\ Berk $^{4}$ \\ 'Department of Medical Pharmacology, Faculty of Medicine, \\ Beykent University, Turkey \\ ${ }^{2}$ Department of Pharmacology, Cerrahpasa Faculty of Medicine, \\ Istanbul University, Turkey \\ ${ }^{3}$ Department of Internal Medicine, Okmeydani Training and \\ Research Hospital, Turkey \\ ${ }^{4}$ Department of Pharmaceutical Chemistry, School of Pharmacy, \\ Istanbul Medipol University, Turkey
}

\begin{abstract}
Correspondence: Gokhan Faikoglu, Department of Medical Pharmacology, Faculty of Medicine, Beykent University, Istanbul, Turkey, Email gokhan.faikoglu@gmail.com
\end{abstract} Received: September 15, 2021 | Published: November 17,
2021
Abbreviations: $\alpha$-GPDH, $\alpha$-glycerophosphate dehydrogenase; LDH, lactate dehydrogenase; NO, nitric oxide; OL, open label; TG, tribenoside + lidocaine combination; SDH, succinate dehydrogenase

\section{Introduction}

Hemorrhoids are the most common proctological condition, especially in sedentary life and during pregnancy. ${ }^{1}$ It has been reported that $36 \%$ of the general population has recurrent hemorrhoids and approximately $50 \%$ has experienced symptomatic hemorrhoids. ${ }^{2}$ The risk of developing hemorrhoids during pregnancy is even higher because of the vascular structure changes with hormonal irregularity, increased intra-abdominal pressure, and constipation. ${ }^{3}$ The prevalence of hemorrhoids persisting after delivery in pregnant women is between $25 \%$ and $35 \%$, and these percentages rise up to $85 \%$ in the third trimester. ${ }^{4}$

The severity of hemorrhoids is classified into 4 clinical grades according to Goligher classification. The medical treatment and lifestyle modifications are convenient for grade I/II hemorrhoids that consist of $>90 \%$ of hemorrhoidal cases. ${ }^{5,6}$ Topical preparations containing anti-inflammatory drugs can be used for medical treatment of hemorrhoids. However steroid-based preparations are associated with the potential onset of adverse events. ${ }^{1,924}$ The combination of tribenoside+lidocaine is a medical preparation for the local treatment of hemorrhoids, delivered as a suppository or rectal cream, have a significant rapid beneficial effect on unpleasant symptoms, such as pain and itching, similar to that of steroid-based preparations. ${ }^{8,24}$

Although tribenoside has a simple saccharide structure, it decreases the secretion of histamine and prostaglandins at the cellular level in the vessel walls in the first 15 minutes after penetration. ${ }^{8,9}$ In the traumatized vessel in hemorrhoids, $\mathrm{NO}$ (nitric oxide) release reaches its maximum level and is sustained with the injury and rupture of the vessel wall. For this reason NO release, is a significant increase in SDH (succinate dehydrogenase) activity, a slight increase in LDH (lactate dehydrogenase) activity, and a significant decrease in $\alpha$-GPDH activity are seen in wall cells. Treatment with tribenoside for 5 days normalizes SDH and $\alpha$-GPDH activity and increases LDH activity. In this way, the vascular wall quickly recovers from the trauma, the tissue begins to repair and restores the functioning to a healthy state..$^{10}$

In addition, tribenoside modulates the expression of laminins, thus helping to restructure the basement membrane of hemorrhoids. ${ }^{11}$ Tribenoside regulates the expression and localization of laminins due to its unique spectrum of pharmacological activities, thus helping to restructure the basement membrane of hemorrhoids has a role in wound healing of hemorrhoids with anti-inflammatory, venotropic, membrane stabilizing, analgesic properties on hemorrhoids. ${ }^{24}$ 
By adding lidocaine to the formulation (tribenoside + lidocaine combination) increases the analgesic effect of tribenoside and provides a rapid local anesthetic action. The synergic pharmacological activity of tribenoside and lidocaine allows to control the inflammatory process and improves local vascular tone, thus decreasing pain and discomfort.

\section{Wound healing properties}

Hemorrhoid is a complex disease involving coagulation, inflammation, and wounds... Although tribenoside has a simple saccharide structure, it inhibits the release of histamine and prostaglandins at the cellular level in the vessel walls in the first 15 minutes after penetration. ${ }^{8,9}$ In the traumatized vessel in hemorrhoids, NO release reaches its maximum level and is sustained with the injury and rupture of the vessel wall. For this NO release, a remarkable increase in succinate dehydrogenase (SDH) activity, a slight increase in lactate dehydrogenase (LDH) activity, and a significant decrease in alpha-glycerophosphate dehydrogenase ( $\alpha$-GPDH) activity are seen in wall cells. Treatment with tribenoside for 5 days normalizes SDH and $\alpha$-GPDH activity, greatly increasing LDH activity. In this way, the vessel wall quickly recovers from the trauma, the tissue begins to repair and restores the functioning to a healthy state. ${ }^{10}$ In addition, studies show that tribenoside interacts with epidermal cells, regulating the expression and localization of laminins, thus helping to restructure the basement membrane in wound healing of hemorrhoids. ${ }^{11}$

Tribenoside, has been indicated in the treatment of hemorrhoids, and presents an efficacy profile not only on the improvement of microcirculation but also wound healing. In human epidermal cells, tribenoside up-regulated the transcription of laminin alpha-5, which is a major component of epidermal bazal membrane. Tribenoside goes into interaction with epidermal cells and manuplates laminins to restructure the bazal membrane during the wound healing process of hemorrhoids. Tribenoside has shown unsurpassed efficacy in the treatment of coagulation, inflammation and wounds. ${ }^{11}$

\section{Overview of clinical evidence}

The effectiveness of tribenoside and lidocaine combination in relieving symptoms caused by hemorrhoids has been assessed in various comparative clinical studies, either versus its two single components (lidocaine and tribenoside) or versus other treatment options belonging to the same therapeutic class. The clinical trials performed with tribenoside+lidocaine combination are reported in Table 1.

Table I Summary of clinical trials with lidocaine and tribenoside combination

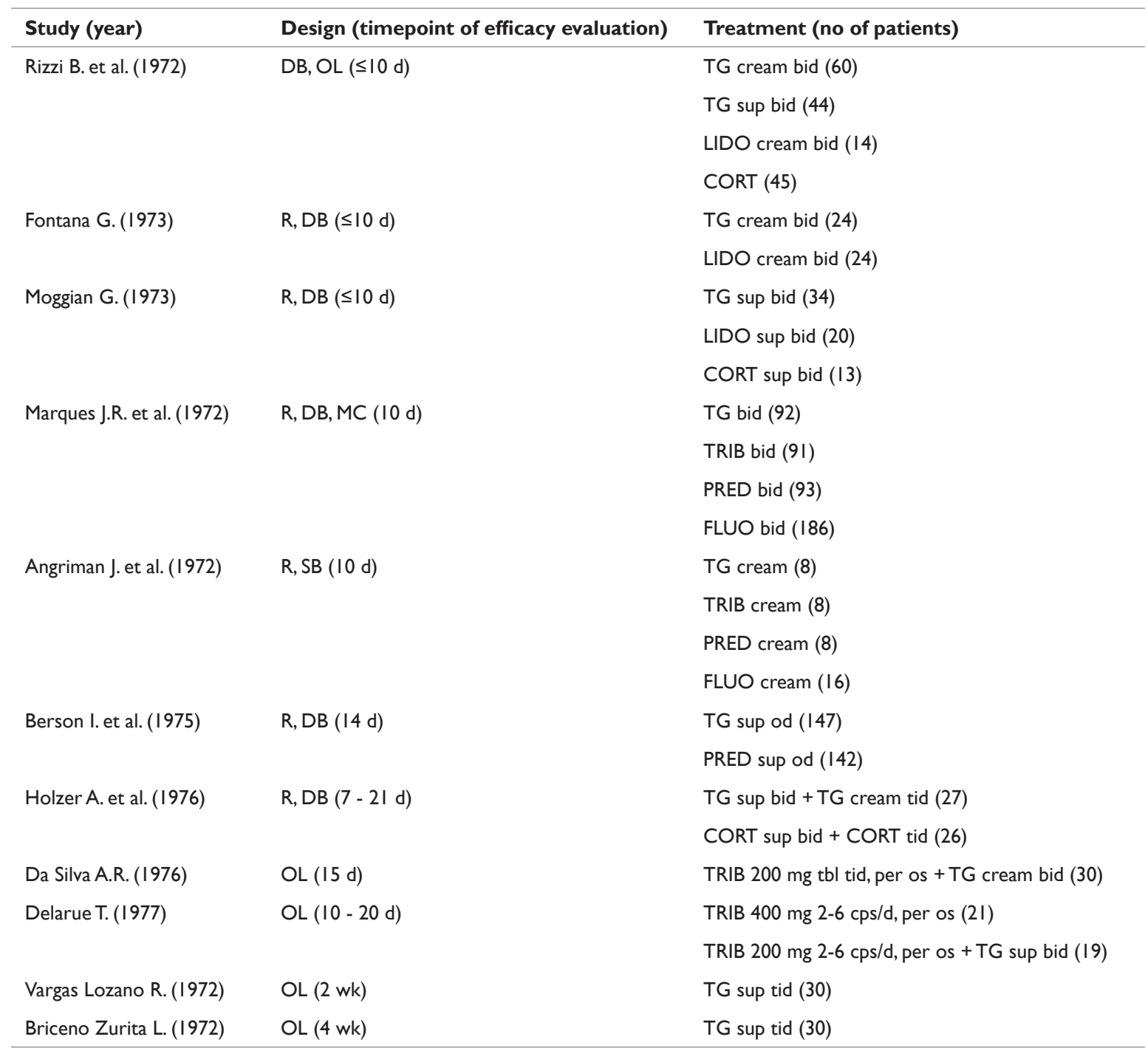

R, randomized; DB, double-blind; SB, single-blind; MC, multicentre; OL, open-label; od, once daily; bid, twice daily, tid, three times a day; TG, tribenoside + lidocaine combination; LIDO, lidocaine; CORT, corticoid-containing prep.;TRIB, tribenoside; PRED, prednisolone-containing prep.; FLUO, fluocinolone-containing prep 
There are 5 studies comparing tribenoside + lidocaine combination (with each of its single components: 3 studies compared tribenoside + lidocaine with a preparation containing only lidocaine (Rizzi et al 1972; Fontana 1973; Moggian 1973). All studies showed the association to be significantly superior to lidocaine alone. Two studies compared tribenoside + lidocaine with a preparation containing only tribenoside (Marques et al 1972; Angriman et al 1972) showing comparable clinical efficacy, although a faster relief of the subjective symptomatology can be claimed by the association.

In 6 studies tribenoside+lidocaine has been compared with a corticosteroid containing preparation (Rizzi, et al 1972; Moggian 1973; Marques et al 1972; Angriman et al 1972; Berson et al 1975, Holzer 1976). In general, tribenoside + lidocaine combination was shown to be at least equally effective to corticosteroids. The long term utilizastion of corticoidsteroids are not recommended due to the possible adverse effects where tribenoside + lidocaine combination can provide sufficient treatment. ${ }^{26}$

Pregnancy cases have been investigated in 2 studies evaluating the effectiveness and tolerability of tribenoside + lidocaine in women with hemorrhoids as a consequence of pregnancy or delivery (Moggian 1973; Delarue 1977), showing good efficacy and favorable tolerability in all enrolled patients.

\section{Description of individual studies}

\section{Rizzi et al. ${ }^{12}$}

Rizzi et al performed an investigation of 4 different studies in patients with hemorrhoids in acute phase to assess the therapeutic activity and tolerability of the tribenoside+lidocaine combination. The first study was a randomized double-blind study comparing tribenoside+lidocaine cream $(n=14)$ with lidocaine $2 \%$ cream $(n=14)$. The 2 nd and 3 th were open-label investigations with tribenoside+lidocaine cream $(n=60)$ and suppositories $(n=44)$ respectively. The 4th study compared tribenoside+lidocaine with steroids-containing preparations (hydrocortisone or triamcinolone or fluocortolone or prednisolone $(n=45)) .^{12}$

In the the comparative studies (1st and $4 t h)$ tribenoside+lidocaine cream performed statistically significant $(p<0.01)$ better results compared to lidocaine and corticosteroid-containing preparations.

In both the open-label studies the activity of tribenoside + lidocaine cream, (60 subjects) and suppositories (44 subjects), applied twice daily for a maximum of 10 days, was evaluated as a) global clinical efficacy (good, mild, ineffective); b) clinical evaluation of objective (secretion, hemorrhage, nodules) and subjective (pain, burning, pruritus) symptoms, using a 4 - points scale $(0=$ absent, $1=$ mild, 2 $=$ moderate, 3 = severe). Both formulations of tribenoside+lidocaine resulted in a rapid subjective and objective symptom improvement with effect on subjective symptoms being already evident before a maximum of 30 minutes since administration. Globally positive results were obtained in $80.7 \%$ of patients.

\section{Fontana et al. ${ }^{13}$}

A double-blind comparative study using a scheme of crossover design was carried out in patients suffering from hemorrhoids. Tribenoside+lidocaine cream was compared with lidocaine 2\% cream. Both preparations were applied twice daily for a maximum of 10 days. ${ }^{13}$ Tribenoside + lidocaine was found to be statistically significant superior $(\mathrm{p}=0.01)$ to lidocaine alone, exerting a clear and rapid effect on both the subjective (pain, itching, sense of weight and tenesmus) and objective (inflammation, hemorrhage, secretion) symptoms. Local and general tolerability was good with no adverse reactions in any of the 48 treated patients. Clinical efficacy was rated as "good" in $93 \%$ of patients assigned to the study combination and only in $28 \%$ of subjects treated only with lidocaine $(p=0.01) .{ }^{13}$

\section{Moggian et al. ${ }^{14}$}

The hemorrhoidal syndrome is often associated with pregnancy and delivery. Tribenoside+lidocaine, was tested in suppository form in 67 subjects in two controlled, double-blind trials: in one trial the experimental preparation was compared with lidocaine, in the other it was compared with a corticosteroid antihemorroidal drug (suppositories containing hydrocortisone 1\%). The clinical efficacy has been evaluated on the objective (secretion, hemorrhage, nodules) and subjective (pain, burning, itching) symptoms according to a 4 -points scale $\left(0=\right.$ absent, $1=$ mild, $2=$ moderate, $3=$ severe. $^{14}$

Tribenoside+lidocaine suppositories significantly improved both subjective (mean score at baseline: 4.62; mean score after treatment: $0.24 ; p<0.01)$ and objective (2.86 vs. 0.91; $p<0.01)$ symptoms of hemorrhoids. Lidocaine only was able to significantly improve $(p<0.01)$ the subjective, but not the objective, symptomatology. ${ }^{14}$

The good clinical efficacy of combination therapy comes from the presence of both lidocaine--with a fast action on subjective symptomsand tribenoside - able to ameliorate objective symptoms and improve vascular tone. ${ }^{14}$

\section{Marques et al. ${ }^{15}$}

A randomized, double-blind, controlled multicenter investigation, carried out in 3 different centers, (Belo Horizonte, Rio de Janeiro and Sao Paulo), by 12 investigators, compared 4 topical proctological preparations, each presented in the 2 galenic forms: cream and suppositories. The study involved 462 patients of both sexes and over 18 years of age, assigned to Tribenoside + lidocaine, either as cream or suppository, tribenoside alone, prednisolone trimethylacetate $0.1 \%$ + vioform $5 \%$ or fuocinolone acetonide $0.01 \%+$ bismuth gallate $5 \%$ + lidocaine $2 \%$. The cream and suppository form were used in 236 and 226 patients respectively. ${ }^{15}$

No statistically significant difference amongst the four studied preparations either cream or suppository was observed on the therapeutic efficacy, which ranged between $84.6 \%$ and $93.5 \%$, respectively. Authors recommended the use of tribenoside+ lidocaine combination as a rapid and effective alternative to corticoid therapy without sharing corticoid-related side effects. ${ }^{15}$

\section{Angriman et al. ${ }^{16}$}

Angriman conducted a single-blind trial on tribenoside+lidocaine cream $(n=8)$, tribenoside $5 \%$ cream $(n=8)$, prednisolone pivalate $0.1 \%+$ vioform $5 \%$ cream $(n=8)$, and fluocinolone acetonide $0.01 \%$ + lidocaine $2 \%$ cream $(n=16)$. Patients were treated for 10 days with the four different topical treatments. Subjective (pruritus, burning, pain) and objective symptoms (secretion, hemorrhage, nodule) were evaluated with a 4 points scale $(0=$ no symptoms, $1=$ mild, $2=$ moderate, 3 = severe); mean total scores for the objective or subjective symptoms were calculated by adding the means of the scores obtained for each symptom. ${ }^{16}$

No significant statistical differences amongst the four treatments were observed. Tribenoside+lidocaine cream showed an effect similar to steroid-containing treatments (fluocinolone, prednisolone), without sharing their potential side-effects. ${ }^{16}$

\section{Berson et al. ${ }^{17}$}

In a double-blind trial in 289 patients with hemorrhoidal symptoms, tribenoside $400 \mathrm{mg}+$ lidocaine $40 \mathrm{mg}$ suppositories $(n=147)$ were compared with prednisolone-containing suppositories 
$(n=142)$. Tribenoside+lidocaine suppositories displayed equal efficacy to the comparative preparation. Particularly evident the potent anti-inflammatory activity and the faster onset of action with tribenoside+lidocaine was confirmed. ${ }^{17}$

\section{Holzer et al. ${ }^{18}$}

This was a randomized, double-blind study that compared local treatment with tribenoside+lidocaine suppositories (tribenoside $400 \mathrm{mg}$ + lidocaine $40 \mathrm{mg}$, b.i.d) and tribenoside+lidocaine cream (tribenoside $5 \% \mathrm{mg}+$ lidocaine $2 \% \mathrm{mg}$, t.i.d) vs a treatment with an hydrocortisone-containing preparation (b.i.d. and .i.d.) cream and suppositories. A total of 53 patients (27 males, 26 females, age: 18-70 years) were treated for 21 days and evaluated at day 7, 14 and 21 . Objective (hemorrhage, secretion, nodules, eczema) and subjective (pain, pruritus, burning) symptoms were assessed with a 4- points scale $(0=$ no symptom, $1=$ mild, $2=$ moderate, $3=$ severe $) .{ }^{18}$

No statistical differences were observed between tribenoside+lidocaine and hydrocortisone treatment. According to the results of this study one can say that the side effects of corticosteroids could be prevented via utilizing tribenoside+lidocaine combination. ${ }^{18}$

\section{Delarue et al. ${ }^{19}$}

The study population consists of 40 women with hemorrhoids during pregnancy $(n=33)$ and delivery $(n=7)$. The patients were administered oral tribenoside $400 \mathrm{mg}$ 2-6 times daily for 10 days (post-partum) or 20 days (pregnancy). Results were rated as excellent in 15, good in 18 and minimal in 7 patients (globally good or very good results in $82.5 \%$ of subjects). Tolerability was favorable in all cases and no systemic or local adverse effects have been observed. ${ }^{19}$

\section{Vargas Lozano et al. ${ }^{20}$}

In this stud 30 patients (16 women, 14 men, mean age: 37.4 years) were administered tribenoside+lidocaine suppositories t.i.d for 2 weeks. The treatment response has been indicated as "excellent" in 11 and "good" in 13 subjects, with $80 \%$ of patients. Many of the symptoms has ameliorated such as pain, bleeding, burning and pruritus. Tolerability has been rated as excellent in all cases. ${ }^{20}$

\section{Da Silva et al. ${ }^{2}$}

In this study protocole 30 patients has received combined treatment with oral tribenoside (200 $\mathrm{mg}$ t.i.d) and local tribenoside+lidocaine cream b.i.d, for 2 weeks. Anal itching has been completely treated in $75 \%$, smarting burning in $72 \%$ and pain in $56 \%$ of subjects. ${ }^{21}$ Globally the results were excellent in $80 \%$ of patients. Tolerability was good in all cases, with no systemic or local adverse effects. ${ }^{21}$

\section{Zurita-Briceno et al. ${ }^{22}$}

This was a single-arm study with 30 patients ( 5 men, mean age: 46 years) who were treated with tribenoside+lidocaine suppositories t.i.d for 4 weeks. Efficacy has been evaluated as "excellent" in 15 $(50 \%)$, "good" in $10(33 \%)$, "moderate" in 4 (13\%) and "insufficient" in $1(3.3 \%)$ patients. The rate of adverse events were very low and seldomly related to treatment. ${ }^{22}$

\section{Discussion}

Hemorrhoids are a widespread condition associated with a number of bothersome symptoms, either subjective (e.g., pain, burning and itching) or objective (e.g., bleeding, swelling and nodules). ${ }^{23}$ For ages the standard treatment was carried out by steroids, but due to the high rate of undesirable effects their preference was limited. There are also some populations with high risk of developing hemorrhoids such as pregnant/breastfeeding women, patients with some infections (e.g., mycoses, $H S V$, local viral infections, $T B C$ ), professional sportsmen or elderly people. ${ }^{24}$

The fixed combination of tribenoside+lidocaine (cream or suppositories) is a single formulation of two different molecules that have complementary and synergic mechanisms of action. Indeed, the combination shows a superior efficacy, compared to its single components, both in subjective and objective symptoms, because tribenoside improves microcirculation, promotes the healing of basement membrane and enhance vascular tone, ${ }^{12,16,24,25}$ while lidocaine, a widely-used local anesthetic, induces a rapid (within 10-30 minutes) relief of subjective symptoms, such as local pain and discomfort, perceived as the most bothersome by patients. ${ }^{12,24}$ Moreover, the combination of tribenoside+lidocaine is at least equally effective as the gold standard treatment for hemorrhoids, i.e., steroidbased preparations, (hydrocortisone, triamcinolone, fluocortolone, or prednisolone) and sometimes superior in providing a prompt relief of the troublesome symptoms, such as pain and itching. Also, tribenoside has a role in wound healing of hemorrhoids with its anti-inflammatory, venotropic, membrane stabilizing and analgesic properties on symptoms of hemorrhoids. ${ }^{24}$ The use of the corticosteroid containing preparations are not recommended for prolonged periods due to the possible steroid-related side-effects, therefore tribenoside + lidocaine can represent a fast, effective and safe medical treatment alternative to these medications. ${ }^{24}$

In the local treatment of hemorrhoids tribenoside+lidocaine combination can be particularly suitable for some patients at high risk to develop hemorrhoids, as elderly, obese and/or sedentary subjects and particularly pregnant women that use of corticosteroids could be contraindicated. Also, tribenoside+lidocaine can be safely administered after the first trimester of pregnancy (although no randomized studies have been conducted in this specific population) and during breastfeeding.

Globally the therapeutic efficacy of tribenoside+lidocaine combination has been evaluated in more than 2500 patients in whom the subjective and objective symptomatology improved within 15 minutes of using the cream or suppositories and completely disappeared within 10 days. ${ }^{12-16}$ Tribenoside+lidocaine cream and suppositories formulation are well tolerated without local or systemic adverse events.

The dual mechanism of tribenoside+lidocaine combination enables faster onset of efficacy on all different symptoms of hemorrhoids while presenting a very good tolerability and safety profile for long term utilization and for pregnancy and elderly. One other important property of the combination can be elaborated as the superior efficacy compared to its single components in improvement of the symptoms of hemorrhoids, likely thanks to its ability to ameliorate both subjective and objective symptoms at the same time. The clinical efficacy on subjective symptoms have been observed within 10-30 minutes in the clinical trials. Also, the efficacy of the tribenoside+lidocaine combination can be stated as long-lasting healing effects on hemorrhoids due to the unique spectrum of pharmacological activities of tribenoside. . $^{16,24,25}$

\section{Summary of evidence}

In clinical and pharmacological terms, the tribenosidelidocaine combination is one of the most striking combinations. The combination is equally effective as steroid-based preparations, which are considered the gold standard treatment for hemorrhoids, in the rapid relief of unpleasant symptoms such as pain and itching. 
However, due to the additional pharmacological properties that tribenoside adds to the formulation, it does not show undesirable side effects on the gastrointestinal system, connective tissue or immune system, and does not affect the prostaglandin synthetase system, unlike corticosteroids or non-steroidal anti-inflammatory agents. ${ }^{8}$

Regarding the mechanistic properties, tribenoside has antiinflammatory, venotropic, membrane stabilizing, weak analgesic and wound healing properties. By adding Lidocaine to the formulation, it was aimed to increase the analgesic effect of tribenoside and to quickly return the patient to his daily routine. Clinical studies with the combination of tribenoside-lidocaine also support the pharmacological findings. In all of the randomized double-blind, single-blind, open and closed-label prospective and retrospective clinical studies conducted with more than 2500 patients, in the evaluations made with subjective and objective criteria as: anal, itching, burning and pain symptoms ameliorate within 15 minutes of using the cream and suppositories and completely disappear within 10 days, and the patient's quality of life improved. ${ }^{12-16}$ No side and/or adverse effects developed in the patients, the safety profile and patient satisfaction rate of the tribenoside + lidocaine combination has been very high. Tribenoside - lidocaine combination had a similar, often superior effect profile to combinations containing placebo, half-placebo with lidocaine, steroids such as hydrocortisone, triamcinolone, fluocortolone, or prednisolone, in all symptoms and healing processes. In addition, it has been determined that it had equal efficacy to steroid+lidocaine, steroid+antibacterial+lidocaine preperations. ${ }^{12-16}$

It is noteworthy that the tribenoside-lidocaine combination may be particularly suitable for certain patient populations at high risk of hemorrhoids for whom steroids may be contraindicated. In particular, it can be safely administered to postpartum women and pregnant women after the first trimester of pregnancy. In addition, the tribenoside+lidocaine combination can also be recommended to athletes for whom rectal steroids are prohibited. ${ }^{26}$

In conclusion, tribenoside + lidocaine represents a rapid, effective and safe option for treatment of hemorrhoids, and should be recommended as first-line treatment of this disease in clinical practice for its long-lasting healing effects on hemorrhoids with high efficacy and good tolerability. ${ }^{26}$

\section{Acknowledgments}

None.

\section{Conflicts of interest}

Gokhan Faikoglu, Kubra Saygisever-Faikoglu and Fatmanur Otmar Ozcan are employees of Recordati.

\section{References}

1. Altomare DF, Giannini I. Pharmacological treatment of hemorrhoids: a narrative review. Expert Opinion on Pharmacotherapy. 2013;14(17):2343-2349.

2. Ganz RA. The evaluation and treatment of hemorrhoids: a guide for the gastroenterologist. Clinical Gastroenterology and Hepatology. 2013;11(6):593-603.

3. Abramowitz L, Sobhani I, Benifla JL, et al. Anal fissure and thrombosed external hemorrhoids before and after delivery. Diseases of the Colon and Rectum. 2002;45(5):650-655.

4. Quijano CE, Abalos E. Conservative management of symptomatic and/ or complicated hemorrhoids in pregnancy and the puerperium. The Cochrane Database of Systematic Reviews. 2005;(3):Cd004077.
5. Kaidar-Person O, Person B, Wexner SD. Hemorrhoidal disease: a comprehensive review. J Am Coll Surg. 2007;204(1):102-117.

6. Riss S, Weiser FA, Schwameis K, et al. The prevalence of hemorrhoids in adults. International Journal of Colorectal Disease. 2012;27(2):215-220.

7. Mounsey AL, Halladay J, Sadiq TS. Hemorrhoids. American Family Physician. 2011;84(2):204-210.

8. Jaques R. The pharmacological activity of tribenoside. Pharmacology. 1977;15(5):445-460.

9. Rüegg M, Jaques R. Tribenoside as an inhibitor of chemically induced histamine release. Experientia. 1974;30(4):399-401.

10. Chaǐka LA, Pankov E. Effect of tribenol on the activity of certain redox enzymes in inflamed venous walls. Farmakologiia $i$ Toksikologiia. 1981;44(1):74-77.

11. Kikkawa Y, Takaki S, Matsuda Y, et al. The influence of Tribenoside on expression and deposition of epidermal laminins in HaCaT cells. Biological \& Pharmaceutical Bulletin. 2010;33(2):307-310.

12. Rizzi B, Giac omini F, Bandini V. Terapia locale della sindrome emorroidaria con etil-3,5,6-tri-o-benzil-d-glicofuranoside: risultati clinici. Gazz Int Med Chirur. 1972;77:1598-1606.

13. Fontana G. Trattamento della sindrome emorroidaria con un derivato glicofuranosidico in crema associato ad un anestetico. Gazz Med Ital. 1973; 132:32-35.

14. Moggian G. Sperimentazione clinica controllata di un derivato glicofuranosidico anti-emorroidario, per uso locale. Minerva Med. 1973;64:215-218.

15. Marques JR, Da Silva JH, Galizia I, et al. Ensaio controlado com Procto-Glyvenol no tratamento das hemorroides. Rev Bras De Cirurgia. 1972;62:7-8.

16. Angriman J, Neumayer F. Tratamiento de las hemorroides con glucofuranosido crema. Orientación Médica. 1972;31:353-354.

17. Berson I, Geiser JD. Utilisation d'un nouveau produit: le proctoglyvenol en pratique proctologique. Revue Suisse de Médecine (Praxis). 1975;63:19-22.

18. Holzer A. Betrachtungen über ano-rektale leidenszustände und ihre behandlung. Der Praktische Arzt. 1976;30:662-672.

19. Delarue T. Traitement de la maladie hemorroidaire pendant la grossesse et le post partum parle glyvenol. Arch Med Ouest. 1977;9:637-641.

20. Vargas Lozano R. Tratamiento sintomatico en hemorroides con glucofuranosidos topicos. Medicina (Rev Mex). 1972;52:575-578.

21. Da Silva A. Doença hemorroidaria-tratamento clinico com o tribenosido. Folha Méd. 1976;73:531-536.

22. Zurita-Briceno L. Tratamiento topico de hemorroides con glucofuranosido. Medicina (Rev Mex). 1972;52:229-232.

23. Trukhan DI, Degovtsov EN, Belkina L. Conservative treatment of hemorrhoids: focus on combination tribenoside and lidocaine. Hospitalreplacing technologies: Ambulatory surgery. 2019;(1-2):106-111.

24. Lorenc Z, Gökçe Ö. Tribenoside and lidocaine in the local treatment of hemorrhoids: an overview of clinical evidence. European Review for Medical and Pharmacological Sciences. 2016;20(12):2742-2751.

25. Summary of Product Characteristics Procto-Glyvenol, $400 \mathrm{mg}+40 \mathrm{mg}$, suppositor Procto-Glyvenol, $5 \%+2 \%$, rectal cream.

26. Kestránek J. Hemorrhoid management in women: the role of tribenoside + lidocaine. Drugs Context. 2019;8:212602. 